\title{
Effects of a Lifestyle Intervention in Routine Care on Short- and Long-Term Maternal Weight Retention and Breastfeeding Behavior-12 Months Follow-up of the Cluster-Randomized GeliS Trial
}

\author{
Julia Hoffmann ${ }^{1} \mathbb{D}^{\mathbb{B}}$, Julia Günther $\left.{ }^{1} \mathbb{(}\right)$, Lynne Stecher ${ }^{1}$, Monika Spies $\left.{ }^{1} \mathbb{(}\right)$, Dorothy Meyer ${ }^{1}{ }^{\mathbb{D}}$, \\ Julia Kunath ${ }^{1}$, Roxana Raab ${ }^{1} \mathbb{D}$, Kathrin Rauh ${ }^{1,2}$ and Hans Hauner ${ }^{1, * \mathbb{D}}$ \\ 1 Else Kröner-Fresenius-Centre for Nutritional Medicine, Klinikum rechts der Isar, Technical University of \\ Munich, Georg-Brauchle-Ring 62, 80992 Munich, Bavaria, Germany; julia.hoffmann@tum.de (J.H.); \\ julia.guenther@tum.de (J.G.); lynne.stecher@tum.de (L.S.); monika.spies@tum.de (M.S.); \\ dora.meyer@tum.de (D.M.); julia.kunath@tum.de (J.K.); roxana.raab@tum.de (R.R.); \\ kathrin.rauh@tum.de (K.R.) \\ 2 Competence Centre for Nutrition (KErn), Am Gereuth 4, 85354 Freising, Bavaria, Germany \\ * Correspondence: hans.hauner@tum.de; Tel.: +49-89-289-249-21
}

Received: 30 April 2019; Accepted: 17 June 2019; Published: 19 June 2019

\begin{abstract}
Postpartum weight retention (PPWR) is associated with an increased risk for maternal obesity and is discussed to be influenced by breastfeeding. The objective was to evaluate the effect of a lifestyle intervention delivered three times during pregnancy and once in the postpartum period on PPWR and on maternal breastfeeding behavior. In total, 1998 participants of the cluster-randomized "healthy living in pregnancy" (GeliS) trial were followed up until the 12th month postpartum (T2 $\mathrm{pp}$ ). Data were collected using maternity records and questionnaires. Data on breastfeeding behavior were collected at $\mathrm{T} 2 \mathrm{pp}$. At $\mathrm{T} 2_{\mathrm{pp}}$, mean PPWR was lower in women receiving counseling (IV) compared to the control group (C) $(-0.2 \pm 4.8 \mathrm{~kg}$ vs. $0.6 \pm 5.2 \mathrm{~kg})$, but there was no significant evidence of between-group differences (adjusted $p=0.123$ ). In the IV, women lost more weight from delivery until $\mathrm{T} 2 \mathrm{pp}$ compared to the $\mathrm{C}$ (adjusted $p=0.008$ ) and showed a slightly higher rate of exclusive breastfeeding (IV: $87.4 \%$; C: $84.4 \%$; adjusted $p<0.001$ ). In conclusion, we found evidence for slight improvements of maternal postpartum weight characteristics and the rate of exclusive breastfeeding in women receiving a lifestyle intervention embedded in routine care, although the clinical meaning of these findings is unclear.
\end{abstract}

Keywords: weight retention; breastfeeding; lifestyle intervention; pregnancy; obesity prevention; routine care; gestational weight gain; postpartum; long-term; follow-up

\section{Introduction}

Obesity prevalence is increasing worldwide and represents one of the major public health concerns [1-3]. In particular, increasing obesity rates are observed in women of childbearing age [4,5]. Moreover, a high pre-pregnancy body mass index (BMI) as well as excessive weight gain during pregnancy are strong contributors to the overall risk of obesity later in life [6-8]. Accordingly, excessive gestational weight gain (GWG) is discussed to be an independent risk factor for both short- and long-term postpartum weight retention (PPWR) [9-12]. Breastfeeding may decrease PPWR, and the duration of breastfeeding has been found to be inversely associated with the woman's risk of overweight [13-15]. Despite this evidence, women with obesity are more likely to fail in breastfeeding and are more susceptible to long-term PPWR and inter-pregnancy weight gain, which contributes to a vicious cycle of obesity progression [16,17]. 
Ultimately, both the prevention of PPWR and the initiation of breastfeeding seem to be key determinants in the prevention of obesity in the postpartum (pp) period. Therefore, various randomized controlled trials (RCTs) offering antenatal and pp lifestyle interventions were conducted to tackle excessive GWG and persistent PPWR as well as to promote breastfeeding. Meta-analyses confirm that antenatal lifestyle interventions are effective in limiting GWG and in reducing long-term maternal PPWR until the 12th month pp $[18,19]$. However, the RCTs included small to moderate numbers of participants, and interventions were usually conducted in community settings and academic facilities [13,20-23]. Approaches embedded in the real-life setting of routine antenatal care are urgently needed to not only assess their effect on excessive GWG but also on long-term PPWR in order to realistically estimate their potential to interfere with the cycle of obesity progression.

The large-scale, cluster-randomized "Gesund leben in der Schwangerschaft" ("healthy living in pregnancy", GeliS) trial was designed as a public health approach embedded in the German antenatal routine care system. The primary aim of the GeliS study was to reduce the proportion of women with excessive GWG according to the weight gain recommendations of the Institute of Medicine (IOM) [24] by offering a comprehensive lifestyle intervention alongside antenatal routine visits [25]. Although the intervention was not effective in limiting excessive GWG [26], it yielded some improvements in dietary (manuscript under revision) and physical activity behavior (manuscript under revision) $[27,28]$. The long-term influence on maternal health remains to be investigated. Herein, our aim was to investigate the effect of the GeliS intervention on short- and long-term maternal PPWR and on the women's breastfeeding behavior assessed in a 12 month follow-up. Moreover, we sought to identify factors influencing long-term PPWR and maternal breastfeeding behavior.

\section{Materials and Methods}

\subsection{The GeliS Study: Design and Setting}

As a prospective, multicenter, cluster-randomized, controlled, open intervention trial, the GeliS study was performed alongside the German routine health care system in five administrative regions in Bavaria. Pairwise cluster-randomization was conducted by randomly matching two districts (clusters) per region according to birth figures and sociodemographic and geographic criteria [25]. This randomization resulted in one control district (C) and one intervention district (IV) per region. Within each of these districts, the study was performed in gynecological and midwifery practices and thus under real-life conditions in the German routine antenatal care system. Further details about study design, setting, and randomization process were described previously [25].

The study was conducted according to current local regulatory requirements and laws as well as in accordance with the declaration of Helsinki. The study protocol was approved by the Ethics Commission of the Technical University of Munich (project number 5653/13) and was retrospectively registered at the ClinicalTrials.gov Protocol Registration System (NCT01958307) [29].

\subsection{Participants and Lifestyle Intervention}

Participants were recruited between 2013 and 2015 in 71 participating gynecological and midwifery practices ( $n=39$ in IV and $n=32$ in C). Eligible were women with (1) a pre-pregnancy BMI between $\geq 18.5 \mathrm{~kg} / \mathrm{m}^{2}$ and $\leq 40.0 \mathrm{~kg} / \mathrm{m}^{2}$, (2) a singleton pregnancy, (3) age between 18 and 43 years, (4) sufficient German language skills and (5) stage of pregnancy before the end of the 12th week of gestation. Before participating in the study, all women gave their written informed consent for inclusion. As described previously, miscarriage or late loss of pregnancy, terminations, pregnancy complications that interfered with the intervention, and maternal death during the course of the trial were considered as exclusion criteria $[25,26]$. During the follow-up, women were defined as drop-outs if they were no longer reachable, did not provide contact details, or withdrew participation. Participants in the $\mathrm{C}$ attended standard antenatal care and obtained only limited information on a healthy antenatal lifestyle and 
the importance of breastfeeding by means of a flyer. The IV received a comprehensive lifestyle intervention program.

The lifestyle intervention program was already described in detail elsewhere [25]. Alongside routine care visits, the IV received three antenatal (12th-16th, 16th-20th, and 30th-34th week of gestation) and one pp (6th-8th week pp) face-to-face counseling sessions lasting between 30 and $45 \mathrm{~min}$, which were given by previously trained midwives, medical personnel, or gynecologists. In these lifestyle sessions, women in the IV were informed about adequate GWG according to the recommendations of the IOM and were encouraged to monitor their weight gain weekly using a weight gain chart [24]. Moreover, healthy dietary and physical activity behavior during pregnancy and the pp period were addressed in accordance with national and international recommendations [30,31]. The importance of breastfeeding for both mothers and their offspring was outlined in both the third antenatal counseling (30th-34th week of gestation) and the pp session (6th-8th week pp). In accordance with German breastfeeding recommendations [32], participants were encouraged to exclusively breastfeed their infants until at least the end of the 4th month after delivery. Furthermore, they were informed about potential barriers to initiate breastfeeding and strategies to overcome these. Additionally, infant hunger and satiety signals were discussed. In case of contraindications of breastfeeding, counselors were advised to refer participants to experts.

Subsequent to the lifestyle intervention, women of both groups underwent the same follow-up observation program with data collection until the 12th month pp. The last participant completed the 12 months follow-up at the end of 2017.

\subsection{Data Collection and Outcomes}

Baseline characteristics were collected using a screening questionnaire at the time of recruitment (before the 12th week of gestation, visit 0 (V0)). Weight was measured in participating gynecological or midwifery practices during the course of pregnancy and 6-8 weeks pp (time point 1 postpartum $\left(\mathrm{T} 1_{\mathrm{pp}}\right)$ ) and was retrieved from maternity records. Weight at the 12th month pp (time point 2 postpartum $\left(\mathrm{T} 2_{\mathrm{pp}}\right)$ ) was self-reported on a questionnaire included in a set of questionnaires ( $\mathrm{QT} 2_{\mathrm{pp}}$, completed at $\mathrm{T} 22_{\mathrm{pp}}$ ). If $\mathrm{QT} 2_{\mathrm{pp}}$ was missing, weight was recorded during a phone interview, which was scheduled at $\mathrm{T} 2 \mathrm{pp}$ with the main purpose of obtaining data on infant anthropometrics. In QT2 $2_{\mathrm{pp}}$, women additionally indicated whether they were pregnant at $\mathrm{T} 2 \mathrm{pp}$.

Pre-pregnancy BMI was calculated based on self-reported pre-pregnancy weight. GWG was defined as the difference between the last measured weight before delivery and the first measured weight at the time of recruitment (V0). Excessive GWG was categorized according to the thresholds provided by the IOM [24]. Short-term PPWR was defined as the difference between the measured weight at 6-8 weeks pp $\left(\mathrm{T}_{\mathrm{pp}}\right)$ and the first measured weight at V0. Long-term PPWR was calculated as the difference between self-reported weight at the 12th month pp $\left(\mathrm{T} 2_{\mathrm{pp}}\right)$ and the first measured weight at V0. For any calculations based on V0 weight, self-reported pre-pregnancy weight was used if the measured V0 weight was missing, which was the case for 27 participants. A PPWR of $5 \mathrm{~kg}$ was shown to be associated with later obesity [33-36]. Therefore, the threshold of $>5 \mathrm{~kg}$ was chosen to define clinically relevant PPWR at $\mathrm{T} 1_{\mathrm{pp}}$ and $\mathrm{T} 2_{\mathrm{pp}}$, respectively.

At $\mathrm{T} 2 \mathrm{pp}$, data on maternal breastfeeding behavior were collected within $\mathrm{QT} 2_{\mathrm{pp}}$ via questions adapted from the German wide survey "German Health Interview and Examination Survey for Children and Adolescents" (KiGGS) [37]. In QT2 2 , women were asked to indicate whether they breastfed their offspring at any time (defined as any breastfeeding) and to estimate the duration of any breastfeeding in months. They additionally stated whether they exclusively breastfed, meaning breastfeeding without offering any formula or complementary food to their infant, and were asked to estimate the duration of exclusive breastfeeding in months. Women were defined as meeting the German breastfeeding recommendations if they reported exclusive breastfeeding until the end of the 4th month pp [32]. 
Data on early antenatal well-being were collected before the 12th week of gestation using the German version of the 5-item World Health Organization Well-Being Index (WHO-5). This is a questionnaire consisting of 5 questions related to the subjective psychological well-being of respondents [38] and has shown to be a valid and reliable indicator of well-being [38,39]. The WHO-5 has been applied across a wide range of study fields, including the field of obstetrics and gynecology, and has been used in comparable clinical trials $[38,40]$. An overall score of less than $50 \%$ has been found to indicate poor well-being and has been used to screen for the diagnosis of depression $[38,39]$. Analyses using the WHO-5 index were performed as described by Topp et al. [38]. Women with a WHO-5 score lower than $50 \%$ were defined as having low antenatal well-being.

Symptoms of postpartum depression (PPD) were assessed at $\mathrm{T} 1_{\mathrm{pp}}$ using the validated German version of the Edinburgh Postnatal Depression Scale (EPDS) [41]. Having an EPDS score of 13 or above was defined as showing symptoms of PPD. Herein, we define PPD as having met the score threshold of the EPDS.

\subsection{Statistical Analysis}

Power calculation was conducted based on the primary study outcome (excessive GWG) and was described elsewhere [25]. All analyses presented herein were performed using SPSS software (IBM SPSS Statistics for Windows, version 24.0, IBM Corp, Armonk, NY, USA).

Analyses for $\mathrm{T} 1_{\mathrm{pp}}$ included all participants except for those who dropped out during the active intervention phase (from inclusion until $\mathrm{T} 1_{\mathrm{pp}}$ ) due to miscarriage or late loss of pregnancy, terminations, pregnancy complications that interfered with the intervention, maternal deaths, or for other reasons (e.g., change of residence or decline of further study participation). Data at $\mathrm{T} 2 \mathrm{pp}$ were reported for all participants without those who were lost to follow-up from $\mathrm{T} 1_{\mathrm{pp}}$ until $\mathrm{T} 2_{\mathrm{pp}}$. PPWR analyses at $\mathrm{T} 2_{\mathrm{pp}}$ additionally excluded all participants that were pregnant.

As defined a priori [26], associations between GWG or excessive GWG and follow-up outcomes were performed as complete-case analyses and included all participants with available GWG except for those who had preterm delivery ( $<37$ th week of gestation) and those who were lost to follow-up.

Baseline characteristics, pp weight characteristics, and breastfeeding information are presented as mean \pm standard deviation (SD) or as proportions if appropriate. Due to the cluster-randomized design, linear regression models and binary logistic regression models fit with generalized estimating equations (GEE) were applied to compare differences between IV and C according to Donner et al. [42]. Group differences are presented as estimated mean difference or odds ratio (OR) with $95 \%$ confidence interval (CI). Adjusted models were controlled for pre-pregnancy BMI category, pre-pregnancy age, and parity. Time variables (gestational age at inclusion, gestational week at birth, $\mathrm{T} 1_{\mathrm{pp}}$ or $\mathrm{T} 2_{\mathrm{pp}}$ ) were controlled as confounders for the corresponding outcome variables. We included exclusive breastfeeding as a further covariate in post-hoc analyses for models related to PPWR. Sensitivity analyses were performed to explore if the inclusion of participants with self-reported pre-pregnancy weight influenced findings on PPWR.

To identify predictors of maternal long-term PPWR and factors influencing exclusive breastfeeding and any breastfeeding, IV and C were pooled to form one cohort. For all cohort analyses, unadjusted linear and binary logistic regression models were applied, as well as models adjusted for pre-pregnancy BMI category, pre-pregnancy age, parity, group assignment, and $\mathrm{T} 2 \mathrm{pp}$. For the outcome long-term PPWR, gestational age at inclusion was included as a further confounding factor.

For all analyses, $p$-values below 0.05 were considered as statistically significant.

\section{Results}

\subsection{Participant Flow and Baseline Characteristics}

In total, 2286 women were enrolled in the GeliS trial (Figure 1). Among them, 25 were not eligible after re-assessment of inclusion criteria, resulting in a total of 2261 participants assigned to 
the IV $(n=1139)$ or the $C(n=1122)$. During the active intervention phase (from recruitment until $\mathrm{T} 1_{\mathrm{pp}}$ ), 263 participants were lost due to miscarriage or late loss of pregnancy (IV: $n=32$; C: $n=41$ ), terminations (IV: $n=3 ; \mathrm{C}: n=6$ ), pregnancy complications that interfered with the intervention (IV: $n=4 ; \mathrm{C}: n=0$ ), or several other reasons (IV: $n=97 ; \mathrm{C}: n=80$ ) such as change of practice/residence, decline of further study participation, and being no longer reachable. Accordingly, 1998 participants (IV: $n=1003$; C: $n=995$ ) remained in the study until $\mathrm{T} 1_{\mathrm{pp}}$. During the course of the follow-up, 101 participants in the IV and 114 in the $C$ were lost to follow-up, as they were not reachable anymore (IV: $n=67$; C: $n=64$ ), did not provide contact details (IV: $n=18 ; \mathrm{C}: n=21$ ), withdrew from study participation (IV: $n=16$; C: $n=28$ ), or due to other reasons (C: $n=1)$, which finally resulted in 1783 participants (IV: $n=902 ; \mathrm{C}: n=881$ ) who provided data for follow-up analyses. The drop-out rate between $\mathrm{T}_{\mathrm{pp}}$ and $\mathrm{T} 2_{\mathrm{pp}}$ was $10.8 \%$. In total, $21.1 \%$ of participants were lost since group allocation.

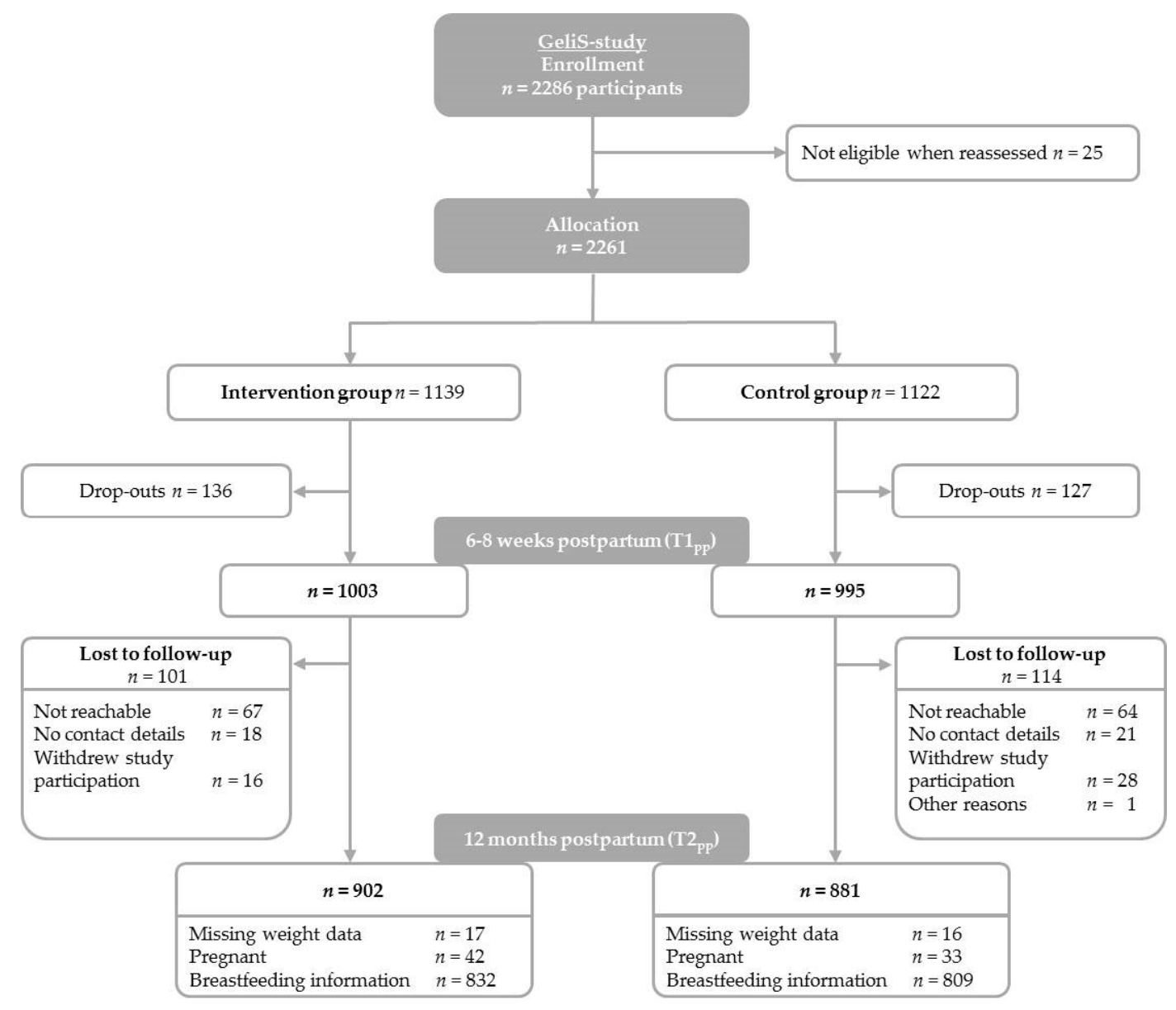

Figure 1. Participant flow.

Baseline characteristics of participants that entered the follow-up are shown in Table 1. Pre-pregnancy weight and BMI were comparable between groups (IV: $68.3 \mathrm{~kg}$ and $24.4 \mathrm{~kg} / \mathrm{m}^{2}$; C: $68.1 \mathrm{~kg}$ and $24.3 \mathrm{~kg} / \mathrm{m}^{2}$ ). In total, $64.9 \%$ of participants showed a BMI between 18.5 and $24.9 \mathrm{~kg} / \mathrm{m}^{2}$ (normal weight), 23.1\% showed a BMI between 25.0 and $29.9 \mathrm{~kg} / \mathrm{m}^{2}$ (overweight), and $12.0 \%$ showed a BMI between 30.0 and $40.0 \mathrm{~kg} / \mathrm{m}^{2}$ (obesity). IV and C were comparable in terms of pre-pregnancy age, GWG, and educational level. As in the active study phase [26], the IV group included more women that were primiparous $(61.8 \%)$ in the pp period compared to the $C(53.2 \%)$. 
Table 1. Baseline characteristics of participants entering the follow-up.

\begin{tabular}{|c|c|c|c|}
\hline & Intervention $(n=1003)$ & Control $(n=995)$ & Total $(n=1998)$ \\
\hline \multicolumn{4}{|l|}{ Maternal characteristics } \\
\hline Pre-pregnancy age, years ${ }^{a}$ & $30.2 \pm 4.3$ & $30.5 \pm 4.6$ & $30.3 \pm 4.4$ \\
\hline Pre-pregnancy weight, $\mathrm{kg}$ & $68.3 \pm 13.0$ & $68.1 \pm 13.7$ & $68.2 \pm 13.4$ \\
\hline Pre-pregnancy BMI, kg/m² & $24.4 \pm 4.3$ & $24.3 \pm 4.6$ & $24.4 \pm 4.5$ \\
\hline \multicolumn{4}{|l|}{ Pre-pregnancy BMI category, $n(\%)$} \\
\hline BMI $18.5-24.9 \mathrm{~kg} / \mathrm{m}^{2}$ & $645 / 1003(64.3 \%)$ & $652 / 995$ (65.5\%) & $1297 / 1998(64.9 \%)$ \\
\hline BMI $25.0-29.9 \mathrm{~kg} / \mathrm{m}^{2}$ & $243 / 1003(24.2 \%)$ & $218 / 995(21.9 \%)$ & $461 / 1998(23.1 \%)$ \\
\hline BMI $30.0-40.0 \mathrm{~kg} / \mathrm{m}^{2}$ & $115 / 1003(11.5 \%)$ & $125 / 995(12.6 \%)$ & $240 / 1998(12.0 \%)$ \\
\hline GWG, kg & $13.9 \pm 5.3$ & $13.9 \pm 5.3$ & $13.9 \pm 5.3$ \\
\hline \multicolumn{4}{|l|}{$\begin{array}{l}\text { GWG classified according to the } \\
\text { IOM, } n(\%)^{\text {b }}\end{array}$} \\
\hline Inadequate GWG & 223/997 (22.4\%) & 201/988 (20.3\%) & $424 / 1985(21.4 \%)$ \\
\hline Adequate GWG & $334 / 997(33.5 \%)$ & $344 / 988(34.8 \%)$ & $678 / 1985(34.2 \%)$ \\
\hline Excessive GWG & $440 / 997(44.1 \%)$ & $443 / 988(44.8 \%)$ & $883 / 1985(44.5 \%)$ \\
\hline GDM, $n(\%)$ & $98 / 984(10.0 \%)$ & $104 / 939(11.1 \%)$ & $202 / 1923(10.5 \%)$ \\
\hline \multicolumn{4}{|l|}{ Educational level } \\
\hline General secondary school & $151 / 1002(15.1 \%)$ & $164 / 992(16.5 \%)$ & $315 / 1994(15.8 \%)$ \\
\hline Intermediate secondary school & $435 / 1002(43.4 \%)$ & $413 / 992(41.6 \%)$ & $848 / 1994(42.5 \%)$ \\
\hline High school & $416 / 1002(41.5 \%)$ & $415 / 992(41.8 \%)$ & $831 / 1994(41.7 \%)$ \\
\hline \multicolumn{4}{|l|}{ Country of birth, $n(\%)$} \\
\hline Germany & $889 / 1003(88.6 \%)$ & $886 / 992(89.0 \%)$ & $1775 / 1995(88.8 \%)$ \\
\hline Others & $114 / 1003(11.4 \%)$ & $106 / 992(10.7 \%)$ & $220 / 1995(11.0 \%)$ \\
\hline Primiparous, $n(\%)$ & $620 / 1003(61.8 \%)$ & $529 / 995(53.2 \%)$ & $1149 / 1998(57.5 \%)$ \\
\hline Smoking in late pregnancy, $n(\%)$ & $41 / 952(4.3 \%)$ & $50 / 938(5.3 \%)$ & $91 / 1890(4.8 \%)$ \\
\hline
\end{tabular}

Abbreviations: GDM: gestational diabetes mellitus; GWG: gestational weight gain; IOM: Institute of Medicine; BMI: body mass index. ${ }^{a}$ mean \pm SD (all such values). ${ }^{b}$ GWG as classified by the IOM [24].

The proportion of participants lost to follow-up was comparable between groups (IV: $10.1 \%$; C: $11.5 \%$ ). Participants who were lost to follow-up differed slightly from women entering the follow-up in terms of pre-pregnancy age, parity, educational level, country of birth, history of gestational diabetes mellitus (GDM), and smoking status during late pregnancy (see Table S1).

\subsection{Weight Outcomes 6-8 Weeks and 12 Months $p p$}

All weight outcomes for the pp period are depicted in Table 2. Women in the IV showed a slightly higher weight at inclusion than in the C (IV: $69.8 \pm 13.1 \mathrm{~kg}$; C: $68.9 \pm 13.9 \mathrm{~kg}$ ). Mean PPWR at 6-8 weeks pp was $4.0 \pm 4.8 \mathrm{~kg}$ in women in the IV and $4.3 \pm 4.8 \mathrm{~kg}$ in women in the C. At T1 $1_{\mathrm{pp}}, 38.2 \%$ of women receiving lifestyle counseling and $40.9 \%$ in the standard care group showed a short-term PPWR above $5 \mathrm{~kg}$ (adjusted $p=0.643$ ). At $\mathrm{T} 1_{\mathrm{pp}}$, there was significant evidence of a difference between groups in women having the same or a lower weight than before pregnancy (IV: $11.3 \%$ vs. C: $14.6 \%$; adjusted $p=0.037)$.

Mean weight loss since delivery until $\mathrm{T} 2 \mathrm{pp}$ was significantly higher in women receiving lifestyle counseling compared to the standard care group (IV: $14.3 \pm 5.9 \mathrm{~kg}$; C: $13.4 \pm 6.0 \mathrm{~kg}$; adjusted effect size $0.85 \mathrm{~kg}, 95 \%$ CI 0.22 to $1.49 ; p=0.008$ ). The mean long-term PPWR was $-0.2 \pm 4.8 \mathrm{~kg}$ for the IV and $0.6 \pm 5.2 \mathrm{~kg}$ for the $C$. In both unadjusted and adjusted models, there was no significant evidence of between-group differences in long-term PPWR related to the first measured weight at inclusion. At T2 $2_{\mathrm{pp}}, 11.4 \%$ of women in the IV and $14.8 \%$ in the $C$ showed a PPWR above $5 \mathrm{~kg}$ (adjusted $p=0.142$ ). Mean PPWR assessed in a per-protocol analysis (data not shown) and the proportion of women at or below the mean pre-pregnancy weight (adjusted $p=0.823$ ) did not differ between groups. At the 12th month pp, $40.5 \%$ of women in the IV and $40.1 \%$ in the C showed a weight at or below pre-pregnancy weight. No difference in PPWR at $\mathrm{T} 1_{\mathrm{pp}}$ and $\mathrm{T} 2_{\mathrm{pp}}$ was found when including participants with the self-reported pre-pregnancy weight if the first measured weight was missing (Table S2). 
Table 2. Weight outcomes in intervention and control groups.

\begin{tabular}{|c|c|c|c|c|c|c|}
\hline & Intervention & Control & $\begin{array}{l}\text { Effect Size }(95 \% \\
\text { CI })\end{array}$ & $p$ & $\begin{array}{l}\text { Adjusted Effect Size } \\
(95 \% \text { CI })\end{array}$ & Adjusted $p$ \\
\hline \multicolumn{7}{|l|}{$\begin{array}{c}\text { Maternal weight } \\
\text { characteristics }\end{array}$} \\
\hline First measured weight, $\mathrm{kg}^{\mathrm{a}, \mathrm{b}}$ & $69.8 \pm 13.1$ & $68.9 \pm 13.9$ & & & & \\
\hline Weight $\mathrm{T} 1_{\mathrm{pp}}, \mathrm{kg}^{\mathrm{b}}$ & $73.8 \pm 13.4$ & $73.0 \pm 13.7$ & $0.62(-1.10,2.35)$ & 0.479 & $0.53(-0.32,1.39)^{\mathrm{d}}$ & $0.221^{\mathrm{d}}$ \\
\hline Weight $\mathrm{T} 2 \mathrm{pp}, \mathrm{kg}^{\mathrm{c}}$ & $69.7 \pm 13.7$ & $69.8 \pm 14.4$ & $-0.26(-2.10,1.58)$ & 0.783 & $-0.04(-0.97,0.89)^{d}$ & $0.935^{\mathrm{d}}$ \\
\hline \multicolumn{7}{|l|}{ Short-term PPWR-T1 $1_{p p} b$} \\
\hline WL since delivery, $\mathrm{kg}^{\mathrm{e}}$ & $9.9 \pm 3.4$ & $9.7 \pm 3.4$ & $0.20(-0.15,0.56)$ & 0.267 & $0.11(-0.22,0.44)^{\mathrm{f}}$ & $0.500^{f}$ \\
\hline PPWR, $\mathrm{kg}$ & $4.0 \pm 4.8$ & $4.3 \pm 4.8$ & $-0.12(-0.94,0.71)$ & 0.783 & $\begin{array}{l}-0.16(-0.95,0.63)^{d} \\
-0.14(-0.96,0.68)^{g}\end{array}$ & $\begin{array}{l}0.694^{\mathrm{d}} \\
0.742^{\mathrm{g}}\end{array}$ \\
\hline PPWR > 5 kg, $n(\%)$ & $\begin{array}{l}372 / 973 \\
(38.2 \%)\end{array}$ & $\begin{array}{l}382 / 934 \\
(40.9 \%)\end{array}$ & $0.95(0.71,1.28)$ & 0.728 & $0.94(0.71,1.24)^{\mathrm{d}}$ & $0.643^{\mathrm{d}}$ \\
\hline $\begin{array}{c}\text { Women } \leq \text { pre-pregnancy } \\
\text { weight, } n(\%)\end{array}$ & $\begin{array}{l}110 / 973 \\
(11.3 \%)\end{array}$ & $\begin{array}{l}136 / 934 \\
(14.6 \%)\end{array}$ & $0.75(0.56,1.01)$ & 0.055 & $0.74(0.56,0.98)^{h}$ & $0.037^{\mathrm{h}}$ \\
\hline \multicolumn{7}{|l|}{ Long-term PPWR-T2 $2_{p p}{ }^{c}$} \\
\hline WL since delivery, $\mathrm{kg}^{\mathrm{e}}$ & $14.3 \pm 5.9$ & $13.4 \pm 6.0$ & $0.86(0.21,1.51)$ & 0.009 & $0.85(0.22,1.49)^{\mathrm{f}}$ & $0.008^{\mathrm{f}}$ \\
\hline PPWR, $\mathrm{kg}$ & $-0.2 \pm 4.8$ & $0.6 \pm 5.2$ & $-0.63(-1.44,0.19)$ & 0.132 & $\begin{array}{l}-0.69(-1.57,0.19)^{d} \\
-0.74(-1.55,0.07)^{g}\end{array}$ & $\begin{array}{l}0.123^{\mathrm{d}} \\
0.075^{\mathrm{g}}\end{array}$ \\
\hline PPWR > 5 kg, $n(\%)$ & $\begin{array}{c}96 / 843 \\
(11.4 \%)\end{array}$ & $\begin{array}{l}123 / 832 \\
(14.8 \%)\end{array}$ & $0.81(0.55,1.19)$ & 0.277 & $0.72(0.47,1.11)^{\mathrm{d}}$ & $0.142^{d}$ \\
\hline $\begin{array}{c}\text { Women } \leq \text { pre-pregnancy } \\
\text { weight, } n(\%)\end{array}$ & $\begin{array}{l}341 / 843 \\
(40.5 \%)\end{array}$ & $\begin{array}{l}334 / 832 \\
(40.1 \%)\end{array}$ & $1.02(0.84,1.23)$ & 0.876 & $1.02(0.84,1.25)^{\mathrm{h}}$ & $0.823^{h}$ \\
\hline
\end{tabular}

Abbreviations: GEE: generalized estimating equations; $\mathrm{T} 1_{\mathrm{pp}}: 6-8$ weeks $\mathrm{pp} ; \mathrm{T} 2_{\mathrm{pp}}$ : 12th month pp; $p$ : $p$ value; pp: postpartum; PPWR: pp weight retention; WL: weight loss. ${ }^{a}$ Mean \pm SD (all such values). ${ }^{b}$ Included are all women with available weight data for $\mathrm{T} 1_{\mathrm{pp}}$ (IV: $n=973$; C: $\left.n=934\right)$. ${ }^{\mathrm{c}}$ Excluded are pregnant women $(n=75)$ and women lost to follow-up $(n=215)$. ${ }^{\mathrm{d}}$ Linear or binary logistic regression models fit using GEEs adjusted for pre-pregnancy BMI, pre-pregnancy age, parity, gestational age at inclusion, time of the pp weight assessment $\left(\mathrm{T} 1_{\mathrm{pp}}\right.$ or $\left.\mathrm{T} 2_{\mathrm{pp}}\right)$. ${ }^{\mathrm{e}}$ Included are all women with available weight data at delivery and pp (T1 $1_{\mathrm{pp}}: \mathrm{IV}: n=970$; C: $n=929$; T2 ${ }_{p p}$ IV: $\left.n=841 ; \mathrm{C}: n=828\right) .{ }^{\mathrm{f}}$ Linear or binary logistic regression models fit using GEEs adjusted for pre-pregnancy BMI, pre-pregnancy age, parity, time of the weight assessment (gestational week at birth and $\mathrm{T}_{\mathrm{pp}}$ or $\mathrm{T} 2$ pp). ${ }^{g}$ Post-hoc adjustment for exclusive breastfeeding. ${ }^{\mathrm{h}}$ Linear or binary logistic regression models fit using GEEs adjusted for pre-pregnancy BMI, pre-pregnancy age, parity, time of the pp weight assessment $\left(\mathrm{T} 1_{\mathrm{pp}}\right.$ or $\left.\mathrm{T} 2_{\mathrm{pp}}\right)$.

\subsection{Breastfeeding Behavior}

Table 3 depicts characteristics of breastfeeding behavior. Any breastfeeding during the pp period was reported by $84.7 \%$ of women in the IV and $85.2 \%$ in the C (adjusted $p=0.954$ ). Among breastfeeding women, the proportion of exclusive breastfeeding differed significantly between groups in unadjusted and adjusted models (IV: 87.4\%; C: 84.4\%; adjusted OR 1.49, 95\% CI 1.22 to $1.81 ; p<0.001$ ). Women in the IV breastfed for $6.6 \pm 3.3$ months on average and breastfed exclusively for $4.8 \pm 1.8$ months on average, whereas women in the $C$ breastfed for $6.4 \pm 3.2$ months on average and breastfed exclusively for $4.7 \pm 1.7$ months on average. There was no significant evidence of between-group differences in breastfeeding duration, the proportion of women who breastfed longer than 12 months, or the proportion of women who met breastfeeding recommendations (Table 3).

Table 3. Breastfeeding behavior of women in intervention and control groups.

\begin{tabular}{|c|c|c|c|c|c|c|}
\hline & $\begin{array}{c}\text { Intervention } \\
n(\%)\end{array}$ & $\begin{array}{l}\text { Control } n \\
(\%)\end{array}$ & Effect Size $(95 \%$ CI) & $p$ & $\begin{array}{l}\text { Adjusted Effect } \\
\text { Size }^{b}(95 \% \text { CI })\end{array}$ & Adjusted $p^{\mathrm{b}}$ \\
\hline \multicolumn{7}{|l|}{ Breastfeeding behavior } \\
\hline \multicolumn{7}{|l|}{ Any breastfeeding } \\
\hline No & $\begin{array}{l}127 / 828 \\
(15.3 \%)\end{array}$ & $\begin{array}{l}119 / 804 \\
(14.8 \%)\end{array}$ & & & & \\
\hline Yes & $\begin{array}{l}701 / 828 \\
(84.7 \%)\end{array}$ & $\begin{array}{l}685 / 804 \\
(85.2 \%)\end{array}$ & $0.95(0.50,1.81)$ & 0.887 & $0.98(0.55,1.76)$ & 0.954 \\
\hline $\begin{array}{l}\text { Breastfeeding duration, } \\
\text { months }{ }^{\mathrm{a}, \mathrm{c}}\end{array}$ & $6.6 \pm 3.3$ & $6.4 \pm 3.2$ & $0.23(-0.15,0.60)$ & 0.236 & $0.23(-0.07,0.54)$ & 0.135 \\
\hline Breastfeeding $\geq 12$ months & $\begin{array}{l}216 / 701 \\
(30.8 \%)\end{array}$ & $\begin{array}{l}183 / 685 \\
(26.7 \%)\end{array}$ & $1.22(0.82,1.83)$ & 0.331 & $1.33(0.89,1.99)$ & 0.170 \\
\hline
\end{tabular}


Table 3. Cont.

\begin{tabular}{|c|c|c|c|c|c|c|}
\hline & $\begin{array}{c}\text { Intervention } \\
n(\%)\end{array}$ & $\begin{array}{l}\text { Control } n \\
(\%)\end{array}$ & Effect Size $(95 \%$ CI) & $p$ & $\begin{array}{l}\text { Adjusted Effect } \\
\text { Size }^{b}(95 \% \text { CI })\end{array}$ & Adjusted $p^{\mathrm{b}}$ \\
\hline \multicolumn{7}{|l|}{ Exclusive breastfeeding } \\
\hline No & $85 / 673(12.6)$ & $\begin{array}{c}103 / 661 \\
(15.6)\end{array}$ & & & & \\
\hline Yes & $\begin{array}{l}588 / 673 \\
(87.4 \%)\end{array}$ & $\begin{array}{l}558 / 661 \\
(84.4 \%)\end{array}$ & $1.32(1.05,1.67)$ & 0.019 & $1.49(1.22,1.81)$ & $<0.001$ \\
\hline $\begin{array}{l}\text { Duration of exclusive } \\
\text { breastfeeding, months a }\end{array}$ & $4.8 \pm 1.8$ & $4.7 \pm 1.7$ & $0.09(-0.05,0.24)$ & 0.216 & $0.15(-0.02,0.32)$ & 0.075 \\
\hline $\begin{array}{l}\text { Meeting breastfeeding } \\
\text { recommendations } \mathrm{d}\end{array}$ & $\begin{array}{l}476 / 587 \\
(81.1 \%)\end{array}$ & $\begin{array}{l}446 / 553 \\
(80.7 \%)\end{array}$ & $1.08(0.95,1.23)$ & 0.232 & $1.08(0.94,1.24)$ & 0.267 \\
\hline
\end{tabular}

Abbreviations: $p$ : $p$ value; $\mathrm{T}_{\mathrm{pp}}$ : 12th month postpartum. ${ }^{\mathrm{a}}$ Mean $\pm \mathrm{SD}$ (all such values). ${ }^{\mathrm{b}}$ Adjusted for maternal pre-pregnancy age, pre-pregnancy BMI, parity, $\mathrm{T} 2$ pp $\cdot{ }^{\mathrm{c}}$ Without participants that still breastfeed. ${ }^{\mathrm{d}}$ Included are participants that exclusively breastfed. Breastfeeding recommendations are defined according to [32].

\subsection{Factors Influencing Long-Term PPWR and Maternal Breastfeeding Behavior}

In the GeliS cohort, we found significant evidence for a positive association of PPWR at $\mathrm{T} 2 \mathrm{pp}$ with pre-pregnancy BMI category (Table 4; adjusted $p=0.012$ ). Age categories were shown to significantly inversely influence PPWR at T2 2 (Table 4 ; adjusted $p=0.013$ ) as did educational level (adjusted $p=0.001$ ), with women of the highest educational level showing the lowest PPWR compared to women with the lowest educational level (adjusted $p=0.001$ ). There was significant evidence of an association between long-term PPWR and excessive GWG (adjusted $p<0.001$ ), parity (adjusted $p=0.007$ ) and the binary outcome of primiparity before study entry (adjusted $p=0.002$ ), and relevant PPWR at $\mathrm{T} 1_{\mathrm{pp}}$ (adjusted $p<0.001$ ) and exclusive breastfeeding (adjusted $p=0.005$ ), but not between PPWR at T2 pp and any breastfeeding, antenatal well-being, or PPD (Table 4). Apart from exclusive breastfeeding (adjusted $p=0.012$ ), no interaction with group assignment was found.

Table 4. Predictors of long-term PPWR.

\begin{tabular}{|c|c|c|c|c|}
\hline PPWR at $T 2_{p p}$ & Effect Size (95\% CI) & $p$ & $\begin{array}{l}\text { Adjusted Effect } \\
\text { Size }(95 \% \text { CI })\end{array}$ & Adjusted $p$ \\
\hline Pre-pregnancy BMI category ${ }^{a}$ & & 0.021 & & 0.012 \\
\hline BMI $18.5-24.9 \mathrm{~kg} / \mathrm{m}^{2}$ & Reference & & Reference & \\
\hline BMI $25.0-29.9 \mathrm{~kg} / \mathrm{m}^{2}$ & $0.47(-0.10,1.05)$ & 0.106 & $1.01(0.28,1.74)$ & 0.006 \\
\hline BMI $30.0-40.0 \mathrm{~kg} / \mathrm{m}^{2}$ & $-0.72(-1.48,0.03)$ & 0.060 & $0.44(-0.82,1.70)$ & 0.494 \\
\hline Age categories (pre-pregnancy) ${ }^{b}$ & & 0.002 & & 0.013 \\
\hline $18-25$ years & Reference & & Reference & \\
\hline $26-35$ years & $-1.29(-2.04,-0.55)$ & 0.001 & $-1.13(-1.89,-0.38)$ & 0.003 \\
\hline $36-43$ years & $-1.39(-2.34,-0.44)$ & 0.004 & $-1.00(-1.98,-0.02)$ & 0.045 \\
\hline Educational level $^{\mathrm{c}}$ & & 0.001 & & 0.001 \\
\hline General secondary school & Reference & & Reference & \\
\hline Intermediate secondary school & $-0.45(-1.18,0.27)$ & 0.221 & $-0.57(-1.29,0.16)$ & 0.129 \\
\hline High school & $-1.21(-1.93,-0.48)$ & 0.001 & $-1.28(-2.02,-0.54)$ & 0.001 \\
\hline $\begin{array}{l}\text { Excessive GWG according to the } \\
\text { IOM }^{\text {c,d }}\end{array}$ & & $<0.001$ & & $<0.001$ \\
\hline Non-excessive GWG & Reference & & Reference & \\
\hline Excessive GWG & $2.04(1.56,2.53)$ & $<0.001$ & $2.25(1.74,2.77)$ & $<0.001$ \\
\hline Parity ${ }^{e}$ & & 0.002 & & 0.002 \\
\hline Primiparous & Reference & & Reference & \\
\hline Multiparous & $0.77(0.29,1.25)$ & 0.002 & $0.77(0.27,1.27)$ & 0.002 \\
\hline
\end{tabular}


Table 4. Cont.

\begin{tabular}{|c|c|c|c|c|}
\hline PPWR at $T 2_{p p}$ & Effect Size $(95 \%$ CI) & $p$ & $\begin{array}{c}\text { Adjusted Effect } \\
\text { Size }(95 \% \text { CI })\end{array}$ & Adjusted $p$ \\
\hline PPWR at $\mathrm{T} 1_{\mathrm{pp}}{ }^{\mathrm{c}}$ & & $<0.001$ & & $<0.001$ \\
\hline PPWR $\leq 5$ kg & Reference & & Reference & \\
\hline PPWR > $5 \mathrm{~kg}$ & $3.84(3.38,4.30)$ & $<0.001$ & $3.82(3.36,4.28)$ & $<0.001$ \\
\hline Any breastfeeding ${ }^{c}$ & & 0.295 & & 0.280 \\
\hline No & Reference & & Reference & \\
\hline Yes & $0.37(-0.32,1.05)$ & 0.295 & $0.38(-0.31,1.06)$ & 0.280 \\
\hline Exclusive breastfeeding $^{c}$ & & 0.002 & & 0.005 \\
\hline No & Reference & & Reference & \\
\hline Yes & $-1.24(-2.02,-0.46)$ & 0.002 & $-1.13(-1.92,-0.33)$ & 0.005 \\
\hline Antenatal well-being $^{c}$ & & 0.447 & & 0.500 \\
\hline Moderate to high & Reference & & Reference & \\
\hline Low & $0.20(-0.31,0.71)$ & 0.447 & $0.18(-0.33,0.69)$ & 0.500 \\
\hline PPD $^{c}$ & & 0.261 & & 0.268 \\
\hline No & Reference & & Reference & \\
\hline Yes & $0.51(-0.38,1.40)$ & 0.261 & $0.50(-0.38,1.38)$ & 0.268 \\
\hline
\end{tabular}

Depicted is the regression coefficient of the PPWR in $\mathrm{kg}$ at $\mathrm{T} 2_{\mathrm{pp}}$ along with the $95 \%$ confidence interval (CI). Abbreviations: GWG: Gestational weight gain; IOM: Institute of Medicine; $p: p$ value; pp: postpartum; PPD: pp depression; PPWR: pp weight retention; $\mathrm{T} 1_{\mathrm{pp}}$ : 6-8 weeks pp; $\mathrm{T} 2_{\mathrm{pp}}$ : 12 th month $\mathrm{pp} .{ }^{\mathrm{a}}$ Linear regression model adjusted for pre-pregnancy age, parity, group assignment, gestational age at inclusion, $\mathrm{T} 2 \mathrm{pp} .{ }^{\mathrm{b}}$ Linear regression model adjusted for pre-pregnancy BMI, parity, group assignment, gestational age at inclusion, $\mathrm{T} 2 \mathrm{pp}$. ${ }^{\mathrm{C}}$ Linear regression model adjusted for pre-pregnancy BMI, pre-pregnancy age, parity, group assignment, gestational age at inclusion, $\mathrm{T} 2 \mathrm{pp} .{ }^{\mathrm{d}}$ Excessive GWG as defined by the IOM [24]. ${ }^{\mathrm{e}}$ Linear regression model adjusted for pre-pregnancy BMI, pre-pregnancy age, group assignment, gestational age at inclusion, $\mathrm{T} 2 \mathrm{pp}$.

Table 5 depicts factors that influenced whether a woman chose to exclusively breastfeed, and Table S3 reports variables that influenced if a woman provided any breastfeeding. Pre-pregnancy BMI category was significantly associated with both any breastfeeding (Table S3; adjusted $p=0.002$ ) and exclusive breastfeeding (Table 5; adjusted $p=0.001$ ), showing that women with a BMI between 25.0 and $29.9 \mathrm{~kg} / \mathrm{m}^{2}$ (adjusted $p=0.006$ ) or a BMI between 30.0 and $40.0 \mathrm{~kg} / \mathrm{m}^{2}$ (adjusted $p=0.002$ ) were less likely to breastfeed exclusively compared to women with normal weight (BMI between 18.5 and $24.9 \mathrm{~kg} / \mathrm{m}^{2}$ ). There was significant evidence for an association between educational level and any breastfeeding (Table S3; adjusted $p<0.001$ ) and exclusive breastfeeding (Table 5; adjusted $p=0.024$ ), with women with a higher educational level being more likely to adopt the respective breastfeeding behavior. Both antenatal well-being and PPD significantly decreased the odds of any breastfeeding (Table S3; adjusted $\mathrm{p}$ value for antenatal well-being $p=0.018$ and PPD $p<0.001$, respectively) but not of exclusive breastfeeding. There was no evidence of excessive GWG influencing either of the breastfeeding outcomes (Table 5; Table S3).

Table 5. Predictors of exclusive breastfeeding.

\begin{tabular}{|c|c|c|c|c|c|c|c|c|}
\hline & \multicolumn{2}{|c|}{$\begin{array}{c}\text { Exclusive } \\
\text { Breastfeeding }\end{array}$} & \multicolumn{2}{|c|}{$\begin{array}{l}\text { No Exclusive } \\
\text { Breastfeeding }\end{array}$} & \multirow{2}{*}{$\begin{array}{l}\text { Effect Size } \\
(95 \% \mathrm{CI})\end{array}$} & \multirow[t]{2}{*}{$p$} & \multirow{2}{*}{$\begin{array}{l}\text { Adjusted } \\
\text { Effect Size } \\
(95 \% \mathrm{CI})\end{array}$} & \multirow[t]{2}{*}{ Adjusted $p$} \\
\hline & $n$ & $\%$ & $n$ & $\%$ & & & & \\
\hline BMI $18.5-24.9 \mathrm{~kg} / \mathrm{m}^{2}$ & $781 / 885$ & 88.2 & $104 / 885$ & 11.8 & Reference & & Reference & \\
\hline BMI $25.0-29.9 \mathrm{~kg} / \mathrm{m}^{2}$ & $253 / 306$ & 82.7 & $53 / 306$ & 17.3 & $0.64(0.44,0.91)$ & 0.014 & $0.60(0.41,0.87)$ & 0.006 \\
\hline $\begin{array}{c}\text { Age categories } \\
\text { (pre-pregnancy) }\end{array}$ & & & & & & 0.725 & & 0.124 \\
\hline $18-25$ years & $131 / 151$ & 86.8 & $20 / 151$ & 13.2 & Reference & & Reference & \\
\hline 26-35 years & $873 / 1013$ & 86.2 & $140 / 1013$ & 13.8 & $0.95(0.58,1.58)$ & 0.848 & $0.81(0.49,1.36)$ & 0.430 \\
\hline $36-43$ years & $142 / 169$ & 84 & $27 / 169$ & 16.0 & $0.80(0.43,1.50)$ & 0.491 & $0.53(0.28,1.02)$ & 0.057 \\
\hline
\end{tabular}


Table 5. Cont.

\begin{tabular}{|c|c|c|c|c|c|c|c|c|}
\hline & \multicolumn{2}{|c|}{$\begin{array}{c}\text { Exclusive } \\
\text { Breastfeeding }\end{array}$} & \multicolumn{2}{|c|}{$\begin{array}{l}\text { No Exclusive } \\
\text { Breastfeeding }\end{array}$} & \multirow{2}{*}{$\begin{array}{c}\text { Effect Size } \\
(95 \% \text { CI })\end{array}$} & \multirow[t]{2}{*}{$p$} & \multirow{2}{*}{$\begin{array}{c}\text { Adjusted } \\
\text { Effect Size } \\
(95 \% \text { CI })\end{array}$} & \multirow{2}{*}{ Adjusted $p$} \\
\hline & $n$ & $\%$ & $n$ & $\%$ & & & & \\
\hline General secondary school & $113 / 144$ & 78.5 & $31 / 144$ & 21.5 & Reference & & Reference & \\
\hline $\begin{array}{l}\text { Intermediate secondary } \\
\text { school }\end{array}$ & $474 / 549$ & 86.3 & $75 / 549$ & 13.7 & $1.73(1.09,2.76)$ & 0.021 & $1.80(1.11,2.92)$ & 0.017 \\
\hline $\begin{array}{l}\text { Excessive GWG according } \\
\text { to the IOM }{ }^{\text {c,d }}\end{array}$ & & & & & & 0.033 & & 0.519 \\
\hline Non-excessive GWG & $612 / 692$ & 88.4 & $80 / 692$ & 11.6 & Reference & & Reference & \\
\hline Excessive GWG & $478 / 567$ & 84.3 & $89 / 567$ & 15.7 & $0.70(0.51,0.97)$ & 0.033 & $0.89(0.62,1.27)$ & 0.519 \\
\hline Antenatal well-being $\mathrm{c}$ & & & & & & 0.337 & & 0.350 \\
\hline No & $1005 / 1164$ & 86.3 & $159 / 1164$ & 13.7 & Reference & & Reference & \\
\hline Yes & $73 / 93$ & 78.5 & $20 / 93$ & 21.5 & $0.58(0.34,0.97)$ & 0.039 & $0.64(0.38,1.10)$ & 0.105 \\
\hline
\end{tabular}

Abbreviations: GWG: Gestational weight gain; IOM: Institute of Medicine; $p: p$ value; pp: postpartum; PPD: pp depression; $\mathrm{T} 2$ pp: 12th month pp. ${ }^{2}$ Binary logistic regression model adjusted for pre-pregnancy age, parity, group assignment, $\mathrm{T} 2 \mathrm{pp} .{ }^{\mathrm{b}}$ Binary logistic regression model adjusted for pre-pregnancy BMI, parity, group assignment, $\mathrm{T} 2 \mathrm{pp} .{ }^{\mathrm{C}}$ Binary logistic regression model adjusted for pre-pregnancy age, pre-pregnancy BMI, parity, group assignment, $\mathrm{T} 2 \mathrm{pp} .{ }^{\mathrm{d}}$ Excessive GWG as defined by the IOM [24].

\section{Discussion}

To our knowledge, this was the first large-scale trial to evaluate the effect of a lifestyle intervention conducted alongside routine care on short- and long-term PPWR and maternal breastfeeding behavior. The GeliS intervention did not improve maternal pp weight development until delivery [26] but yielded some minor improvements in maternal pp weight development and in the proportion of women who exclusively breastfed compared to standard care, although the clinical meaning of these findings is unclear. While the intervention did not seem to influence short-term PPWR and weight loss from delivery until 6-8 weeks pp, mean PPWR at the 12th month pp tended to be lower in the intervention group. Accordingly, weight loss since delivery until the 12th month pp was found to be more pronounced in women who previously obtained counseling. Although a higher proportion of women reached pre-pregnancy weight 6-8 weeks after birth in the control group, this group difference disappeared at the 12th month pp weight measurement. Findings on the influence of pre- and postnatal lifestyle interventions on maternal PPWR are often inconclusive and depend on the definition of PPWR, participant characteristics, and follow-up period, which complicates a comparison of study outcomes. While some studies reported a positive influence of lifestyle interventions on PPWR [20,43], others found no effect [44-46]. A recent meta-analysis suggested a favorable effect of the intervention with an overall weighted mean difference of $-0.73 \mathrm{~kg}(95 \% \mathrm{CI}-1.32$ to $-0.14 ; p=0.015)$ between groups and a weighted mean difference of $-0.68 \mathrm{~kg}(95 \% \mathrm{CI}-1.28$ to $-0.09 ; p=0.023)$ in PPWR 12 months after birth [19]. Although we reported a comparable effect size for long-term PPWR, we found no significant evidence of a between-group difference. This might be explained by the specific study setting of the GeliS trial and the general problem of scaling-up interventions [47]. Unlike GeliS, none of the in the meta-analysis included trials were performed under real-life conditions in the setting of routine antenatal care.

Currently, there is a debate about factors that influence maternal PPWR in the short and the long term and thus might predict the risk for sustained PPWR [48]. We found strong evidence for an association between excessive GWG and long-term PPWR, which was consistently observed by others, confirming excessive GWG as a major risk factor for PPWR even beyond the first year after birth $[9,10,24,44,46,49]$. Moreover, our findings suggest that parity is associated with PPWR, with multiparous women showing a lower PPWR at the 12th month pp compared to primiparous women. Albeit our observations are consistent with reports of others $[48,50]$, the overall opinion on the 
role of parity in PPWR is inconclusive [51]. Similar to others, we reported pre-pregnancy BMI category and educational level to be consistent predictors of long-term PPWR [20,52] and found short-term PPWR $>5 \mathrm{~kg}$ to be strongly associated with PPWR at one year after birth, but not antenatal well-being and PPD. Furthermore, the only breastfeeding pattern we found to beneficially influence PPWR was exclusive breastfeeding, with no observed effect in mothers who practiced any breastfeeding. Some studies confirm a link between breastfeeding and PPWR [13,14,46], while others fail to provide sufficient evidence [20]. This discrepancy might be explained by the heterogeneity observed in methodological procedures and breastfeeding outcomes. Drawing evidence from our findings and relevant literature, the overall effect of breastfeeding on PPWR seems to depend on breastfeeding intensity and duration. We acknowledge that other determinants such as postnatal diet and physical activity behavior may have a fundamental influence on PPWR, and analyzing their contribution on maternal pp weight development might generate ideas for initiatives to prevent long-term PPWR and inter-pregnancy weight gain [53].

Evaluating the success of lifestyle interventions on a woman's risk for sustained weight retention, Sagedal et al. [45] demonstrated a broader spectrum of pp weight characteristics beyond PPWR, addressing variables such as returning to pre-pregnancy weight and weight loss since delivery. Our results showed that weight loss since delivery until the first year after delivery was $14.3 \mathrm{~kg}$ in the IV and $13.4 \mathrm{~kg}$ in the $C$ on average and corresponded to findings by Sagedal et al. (IV: $13.3 \mathrm{~kg}$; C: $14.0 \mathrm{~kg}$ ) [45] and Phelan et al. (IV: $13.6 \mathrm{~kg}$; C: $12.5 \mathrm{~kg}$ ) [20], who included participants from all BMI classes. However, only the GeliS trial showed that women in the IV achieved greater weight loss pp, suggesting the effectiveness of our lifestyle intervention for this outcome. Sagedal et al. reported significant between-group differences in women returning to pre-pregnancy weight (IV: $53.2 \%$; C: $43.1 \%$ ) [45], which is in contrast to results of the "Fit for Delivery" [20] and the GeliS study. Although studies describe effects of lifestyle interventions on pp weight development, its clinical relevance remains to be questioned. PPWRs above $4.55 \mathrm{~kg}$ or $5 \mathrm{~kg}$ are commonly used as markers to define relevant or sustained PPWR and consequently to assess the clinical relevance of PPWR, as weight retention above these thresholds is associated with obesity later in life [24,33-36,54]. Our data did not provide any evidence for an effect of the GeliS intervention on relevant PPWR. Unfortunately, relevant PPWR is only reported in a few other trials [21,43,44,46,55]. Among them, only one study found that lifestyle interventions significantly impacted clinically relevant PPWR at the 12th month after birth [44].

Besides improving maternal weight outcomes in the pp period, the GeliS intervention sought to support participants in the initiation of breastfeeding and showed significant between-group differences in the proportion of women who exclusively breastfed (IV: $87.4 \%$ vs. C: $84.4 \%$; adjusted $p<0.001$ ). However, this difference was rather small, and therefore the clinical meaning of this finding remains unclear. No other breastfeeding outcome appeared to be affected by counseling, which suggests that our intervention was not effective in changing maternal breastfeeding behavior and therefore does not support adoption of our counseling methods in clinical practice. Unfortunately, a comparison with other studies in this field is limited, as few trials included breastfeeding advice or reported relevant data. In a pilot trial that we conducted prior to the GeliS study, we were not able to show between-group differences in the duration of either any breastfeeding or exclusive breastfeeding [44]. Vinter et al. reported similar high numbers of women with obesity initiating breastfeeding in their Danish cohort but no effect of the intervention on breastfeeding outcomes [13]. In the "Fit for Delivery" study, which included women from all BMI classes and did not target breastfeeding behavior, breastfeeding rates were low and did not differ between groups (IV: $10.4 \%$ and C: $8.3 \%$ at the 6 th month pp and $3.4 \%$ and $4.6 \%$, respectively, at the 12 th month pp). Overall, our breastfeeding rates correspond to other German observations that report an increasing trend towards the initiation of any breastfeeding over the last decade $[37,56]$. Similar to our observations, German and US studies found educational level and age before or at birth to be predictive for any breastfeeding [37,57]. Moreover, US observational studies extend our findings, showing that BMI category not only influences breastfeeding initiation but also 
breastfeeding maintenance beyond both the first and the 6th month pp [57]. We found that antenatal well-being and PPD were associated with any breastfeeding but not with exclusive breastfeeding. Others have observed that women with depressive symptoms exclusively breastfed less frequently [58]. Current research expands our findings, reporting inverse associations between antenatal depressive symptoms and the initiation of breastfeeding as well as observations that both antenatal depression and PPD predict early cessation of breastfeeding $[57,59,60]$. Drawing from evidence presented herein, we suggest future initiatives to offer tailored and personalized counseling according to the individual needs of the mother. This will help to elucidate whether antenatal interventions are effective in achieving improvements in maternal breastfeeding behavior.

The analyses presented herein have some limitations. Weight was partly self-reported, which is generally thought to provide valid estimates and is widely used in comparable trials [61,62], but remains subjective. We are aware that weight measurements may not be completely standardized, as data were collected in all participating practices by varying personnel. Moreover, we observed inconsistencies in the first measured weight between groups that we attributed to the timing of the first weight measurement and to between-group differences in participants that were lost to follow-up. To address this limitation, we included "time of the first weight measurement" as a covariate in our analyses on PPWR. Power calculation was conducted based on the primary study endpoint (GWG). The power to detect differences in the secondary outcomes, including PPWR and breastfeeding, was not considered in the study design. We found differences between baseline characteristics among women included in follow-up analyses and those who dropped out in the pp period. Although we adjusted for some of these parameters in our analyses (BMI category and parity), this might have introduced bias and could have influenced results. Counseling was not based on concepts of behavioral change theory, which we acknowledge as a shortcoming related to both the training of counselors and the content of the counseling sessions. Our cohort differed slightly from the average German women of childbearing age in terms of educational level and BMI classes and was therefore not completely representative of the general German population [5]. In order to analyze the effect of GWG on PPWR, we combined women with underweight $(n=38)$ and normal weight to one group. Moreover, we pooled women with inadequate and adequate GWG to one group defined as women with non-excessive GWG. We acknowledge that pooling might have biased the overall influence of the dichotomized outcome excessive GWG on PPWR. We are aware that the contribution of other relevant predictors of PPWR, such as smoking, diet, and physical activity, are not included in our analyses. Moreover, we acknowledge that data on breastfeeding behavior were collected retrospectively by a questionnaire, which may limit its validity.

Notwithstanding our limitations, there are several strengths that merit particular attention. Current research in this field is mainly conducted under controlled conditions in community and academic settings or includes only small to moderate numbers of participants [19]. The GeliS trial was designed as a large-scale lifestyle intervention that was implemented under real-life conditions. Assessing whether a public health intervention is successful and ultimately effective largely depends on its scalability. Through our study, we were able to demonstrate that we could implement a lifestyle intervention in the setting of routine care reaching a wider population of our target group. Findings from this study on pp weight development are promising first steps towards reducing obesity risk of women in the pp period on a broader scale. Herein, we reported minor effects on long-term pp weight development and slight improvements in the rate of exclusive breastfeeding. We were able to assess PPWR in women from all BMI categories and to selectively describe the overall influence of BMI on PPWR and breastfeeding behavior on the cohort level. During the follow-up period, we lost only $10.8 \%$ of participants and in total-since group allocation until the 12th month pp-only $21.1 \%$. This was a rather low drop-out rate for the pp period considering that other studies reported drop-out rates after birth between $15 \%$ and $25 \%[13,20,45]$. We attribute the pp drop-out rate of $10.8 \%$ to our effort of thoroughly informing participants about the importance of the long-term follow-up at study entry. Finally, we reported data on a 12 months follow-up, which was longer than in most other trials and 
contributed valuable information towards estimating the long-term effect of interventions on PPWR. By following participants until the 5th year after birth, we will be able to provide further data on maternal weight development.

\section{Conclusions}

The evidence outlined above demonstrated that the GeliS lifestyle intervention, which was implemented under real-life conditions, yielded some small effects on pp weight development and the rate of exclusive breastfeeding. Moreover, our analysis clearly showed that a considerable proportion of women in both groups retained more than $5 \mathrm{~kg}$ weight at the 12th month pp, which increases their risk of obesity later in life. Additional research will elucidate the effect of the GeliS intervention on PPWR beyond the first year until the 5th year after birth and will be able to evaluate the intervention effect on maternal pp weight development and infant outcomes. Effective strategies embedded in routine care that improve maternal breastfeeding behavior and achieve clinically relevant pp weight outcomes are critical for improving both pre- and postnatal health on a large scale. Tailored interventions that consider different high risk subgroups may increase the success of interventions on PPWR and breastfeeding behavior.

Supplementary Materials: The following are available online at http://www.mdpi.com/2077-0383/8/6/876/s1, Table S1: Characteristics of women entering the follow-up compared to women lost to follow-up, Table S2: Sensitivity analyses on short-term and long-term PPWR, Table S3: Factors influencing the decision to breastfeed (any breastfeeding).

Author Contributions: Conceptualization, H.H., K.R., J.H. and J.G.; methodology, H.H., K.R., J.H., J.G and L.S.; formal analysis, J.H. and L.S.; investigation, J.H., J.G., and J.K.; resources, K.R., J.K. and J.G.; data curation, J.H. and L.S.; writing—original draft preparation, J.H.; writing-review and editing, J.G., K.R., J.K., M.S., D.M., R.R. and H.H.; visualization, J.H.; supervision, H.H.; project administration, K.R., J.H.; funding acquisition, H.H.

Funding: The study is funded by Else Kröner-Fresenius Foundation, Bad Homburg (Grant number: 5140889), the Else Kröner-Fresenius Centre for Nutritional Medicine at the Technical University of Munich, the Competence Centre for Nutrition (KErn) in Bavaria, the Bavarian State Ministry of Food, Agriculture and Forestry, the Bavarian State Ministry of Health and Care (Health Initiative "Gesund.Leben.Bayern."), the AOK Bayern, the largest statutory health insurance in Bavaria, as well as the DEDIPAC consortium by the Joint Programming Initiative (JPI) "A Healthy Diet for a Healthy Life". The funders had no role in the design of the study; in the collection, analyses, or interpretation of data; in the writing of the manuscript, or in the decision to publish the results.

Acknowledgments: We gratefully acknowledge the valuable contribution from our colleagues Eva Rosenfeld and Luzia Kick from the Competence Centre for Nutrition (KErn), the Munich Study Centre at the Technical University of Munich and project managers at the expert centres for nutrition/community catering at the regional offices (AELF) of the Bavarian State Ministry of Food, Agriculture and Forestry (StMELF), which have been coordinating the study on the regional level. We gratefully acknowledge the work and contribution of our colleague Christina Holzapfel, Institute for Nutritional Medicine, Klinikum rechts der Isar, Technical University of Munich. The support of Renate Oberhoffer, Chair of Preventative Pediatrics, Technical University of Munich, Kurt Ulm and Victoria Kehl, Institute of Medical Informatics, Statistics and Epidemiology, Klinikum rechts der Isar of the Technical University of Munich, the network Healthy Start-Young Family Network, Federal Center for Nutrition (BZfE), Federal Office for Agriculture and Food (BLE), belonging to the national IN FORM initiative (Maria Flothkötter, Katharina Krüger), Bonn, Uta Engels, Sports Centre, University of Regensburg, Karl-Heinz Ladwig, Chaterina Sujana and Hamimatunnisa Johar, Research Group Mental Health at the Institute of Epidemiology, Helmholtz Centre Munich, K.T.M. Schneider, Division of Obstetrics and Perinatal Medicine, Technical University of Munich, Rüdiger von Kries, Institute of Social Paediatrics and Adolescent Medicine, Ludwig-Maximilians-University Munich, Regina Ensenauer, von Hauner Children's Hospital, Ludwig-Maximilians-University Munich and Heinrich Heine University Düsseldorf, Rolf Holle, Institute of Health Economics and Health Care Management, Institute of Epidemiology, Helmholtz Centre Munich, Gabi Pfeifer, Educational Center Nuremberg, and Eveline Rieg, Competence Centre for Nutrition, Freising/Kulmbach, is gratefully acknowledged. We are also indebted to the Board of Trustees: Maria Flothkötter, German Federal Ministry of Food and Agriculture (BZfE) in the Federal Office for Agriculture and Food (BLE), Bonn; Beatrix Heilig and Martina Enke, Bavarian State Ministry of Health and Care; Marion Kratzmair and Wolfram Schaecke, Bavarian State Ministry of Food, Agriculture and Forestry; Annette Scheder and Katharina Leopold, AOK Bayern. We would also like to acknowledge the support of the Bayerische Landesärztekammer, Bayerischer Hebammen Landesverband e.V. and the Company Beurer GmbH, Ulm. Finally, we would like to thank our colleagues Kristina Geyer and Annie Naujoks, Institute for Nutritional Medicine, Klinikum rechts der Isar, Technical University of Munich for their support and all participating practices, gynecologists, medical personnel, midwives, participants and their families for their involvement.

Conflicts of Interest: The authors declare no conflict of interest. 


\section{Abbreviations}

\begin{tabular}{|c|c|}
\hline BMI & Body mass index \\
\hline C & Control group \\
\hline CI & Confidence interval \\
\hline EPDS & Edinburgh Postnatal Depression Scale \\
\hline GDM & Gestational diabetes mellitus \\
\hline GEE & Generalized estimating equation \\
\hline GeliS & "Gesund leben in der Schwangerschaft"| "healthy living in pregnancy" \\
\hline GWG & Gestational weight gain \\
\hline $\mathrm{IOM}$ & Institute of Medicine \\
\hline IV & Intervention group \\
\hline KiGGS & German Health Interview and Examination Survey for Children and Adolescents \\
\hline OR & Odds ratio \\
\hline PP & Postpartum \\
\hline PPD & Postpartum depression \\
\hline PPWR & Postpartum weight retention \\
\hline $\mathrm{QT} 2_{\mathrm{pp}}$ & Set of questionnaires provided at $\mathrm{T} 2 \mathrm{pp}$ \\
\hline $\mathrm{RCT}$ & Randomized-controlled trial \\
\hline SD & Standard deviation \\
\hline $\mathrm{T} 1_{\mathrm{pp}}$ & Time point of first data collection postpartum \\
\hline $\mathrm{T} 2 \mathrm{pp}$ & Time point of second data collection postpartum \\
\hline V0 & Visit 0 , time of recruitment (before the 12 th week of gestation) \\
\hline WHO & World Health Organization \\
\hline WHO-5 & 5-item World Health Organization Well-Being Index \\
\hline WL & Weight loss \\
\hline
\end{tabular}

\section{References}

1. Ng, M.; Fleming, T.; Robinson, M.; Thomson, B.; Graetz, N.; Margono, C.; Mullany, E.C.; Biryukov, S.; Abbafati, C.; Abera, S.F.; et al. Global, regional, and national prevalence of overweight and obesity in children and adults during 1980-2013: A systematic analysis for the Global Burden of Disease Study 2013. Lancet 2014, 384, 766-781. [CrossRef]

2. World Health Organization. Obesity and Overweight: Fact sheet No311. Available online: https://www.who. int/news-room/fact-sheets/detail/obesity-and-overweight (accessed on 6 March 2019).

3. Hruby, A.; Hu, F.B. The Epidemiology of Obesity: A Big Picture. Pharmacoeconomics 2015, 33, 673-689. [CrossRef] [PubMed]

4. Balarajan, Y.; Villamor, E. Nationally representative surveys show recent increases in the prevalence of overweight and obesity among women of reproductive age in Bangladesh, Nepal, and India. J. Nutr. 2009, 139, 2139-2144. [CrossRef] [PubMed]

5. Mensink, G.B.M.; Schienkiewitz, A.; Haftenberger, M.; Lampert, T.; Ziese, T.; Scheidt-Nave, C. Übergewicht und Adipositas in Deutschland. Bundesgesundheitsbl. 2013, 56, 786-794. [CrossRef] [PubMed]

6. Rooney, B.L.; Schauberger, C.W.; Mathiason, M.A. Impact of perinatal weight change on long-term obesity and obesity-related illnesses. Obstet. Gynecol. 2005, 106, 1349-1356. [CrossRef] [PubMed]

7. Mamun, A.A.; Kinarivala, M.; O'Callaghan, M.J.; Williams, G.M.; Najman, J.M.; Callaway, L.K. Associations of excess weight gain during pregnancy with long-term maternal overweight and obesity: Evidence from 21 y postpartum follow-up. Am. J. Clin. Nutr. 2010, 91, 1336-1341. [CrossRef]

8. Moll, U.; Olsson, H.; Landin-Olsson, M. Impact of Pregestational Weight and Weight Gain during Pregnancy on Long-Term Risk for Diseases. PLoS ONE 2017, 12, e0168543. [CrossRef]

9. Nehring, I.; Schmoll, S.; Beyerlein, A.; Hauner, H.; Kries, R. von. Gestational weight gain and long-term postpartum weight retention: A meta-analysis. Am. J. Clin. Nutr. 2011, 94, 1225-1231. [CrossRef]

10. Margerison Zilko, C.E.; Rehkopf, D.; Abrams, B. Association of maternal gestational weight gain with shortand long-term maternal and child health outcomes. Am. J. Obstet. Gynecol. 2010, 202, 574.e1-8. [CrossRef] 
11. Mannan, M.; Doi, S.A.R.; Mamun, A.A. Association between weight gain during pregnancy and postpartum weight retention and obesity: A bias-adjusted meta-analysis. Nutr. Rev. 2013, 71, 343-352. [CrossRef]

12. Rong, K.; Yu, K.; Han, X.; Szeto, I.M.Y.; Qin, X.; Wang, J.; Ning, Y.; Wang, P.; Ma, D. Pre-pregnancy BMI, gestational weight gain and postpartum weight retention: A meta-analysis of observational studies. Public Health Nutr. 2015, 18, 2172-2182. [CrossRef] [PubMed]

13. Vinter, C.A.; Jensen, D.M.; Ovesen, P.; Beck-Nielsen, H.; Tanvig, M.; Lamont, R.F.; Jørgensen, J.S. Postpartum weight retention and breastfeeding among obese women from the randomized controlled Lifestyle in Pregnancy (LiP) trial. Acta Obstet. Gynecol. Scand. 2014, 93, 794-801. [CrossRef] [PubMed]

14. Baker, J.L.; Gamborg, M.; Heitmann, B.L.; Lissner, L.; Sørensen, T.I.A.; Rasmussen, K.M. Breastfeeding reduces postpartum weight retention. Am. J. Clin. Nutr. 2008, 88, 1543-1551. [CrossRef] [PubMed]

15. Harder, T.; Bergmann, R.; Kallischnigg, G.; Plagemann, A. Duration of breastfeeding and risk of overweight: A meta-analysis. Am. J. Epidemiol. 2005, 162, 397-403. [CrossRef] [PubMed]

16. Garcia, A.H.; Voortman, T.; Baena, C.P.; Chowdhurry, R.; Muka, T.; Jaspers, L.; Warnakula, S.; Tielemans, M.J.; Troup, J.; Bramer, W.M.; et al. Maternal weight status, diet, and supplement use as determinants of breastfeeding and complementary feeding: A systematic review and meta-analysis. Nutr. Rev. 2016, 74, 490-516. [CrossRef] [PubMed]

17. Christenson, A.; Johansson, E.; Reynisdottir, S.; Torgerson, J.; Hemmingsson, E. Women's Perceived Reasons for Their Excessive Postpartum Weight Retention: A Qualitative Interview Study. PLoS ONE 2016, 11, e0167731. [CrossRef] [PubMed]

18. The International Weight Management in Pregnancy (i-WIP) Collaborative Group. Effect of diet and physical activity based interventions in pregnancy on gestational weight gain and pregnancy outcomes: Meta-analysis of individual participant data from randomised trials. BMJ 2017, 358, j3119. [CrossRef]

19. Michel, S.; Raab, R.; Drabsch, T.; Günther, J.; Stecher, L.; Hauner, H. Do lifestyle interventions during pregnancy have the potential to reduce long-term postpartum weight retention? A systematic review and meta-analysis. Obes. Rev. 2018. [CrossRef]

20. Phelan, S.; Phipps, M.G.; Abrams, B.; Darroch, F.; Grantham, K.; Schaffner, A.; Wing, R.R. Does behavioral intervention in pregnancy reduce postpartum weight retention? Twelve-month outcomes of the Fit for Delivery randomized trial. Am. J. Clin. Nutr. 2014, 99, 302-311. [CrossRef]

21. Vesco, K.K.; Leo, M.C.; Karanja, N.; Gillman, M.W.; McEvoy, C.T.; King, J.C.; Eckhardt, C.L.; Smith, K.S.; Perrin, N.; Stevens, V.J. One-year postpartum outcomes following a weight management intervention in pregnant women with obesity. Obesity 2016, 24, 2042-2049. [CrossRef]

22. McEachan, R.R.C.; Santorelli, G.; Bryant, M.; Sahota, P.; Farrar, D.; Small, N.; Akhtar, S.; Sargent, J.; Barber, S.E.; Taylor, N.; et al. The HAPPY (Healthy and Active Parenting Programmme for early Years) feasibility randomised control trial: Acceptability and feasibility of an intervention to reduce infant obesity. BMC Public Health 2016, 16, 211. [CrossRef] [PubMed]

23. Peccei, A.; Blake-Lamb, T.; Rahilly, D.; Hatoum, I.; Bryant, A. Intensive Prenatal Nutrition Counseling in a Community Health Setting: A Randomized Controlled Trial. Obstet. Gynecol. 2017, 130, 423-432. [CrossRef] [PubMed]

24. National Research Council. Weight Gain During Pregnancy. Reexamining the Guidelines; Rasmussen, K.M., Yaktine, A.L., Eds.; National Academy of Sciences: Washington, DC, USA, 2009; ISBN 9780309131131.

25. Rauh, K.; Kunath, J.; Rosenfeld, E.; Kick, L.; Ulm, K.; Hauner, H. Healthy living in pregnancy: A cluster-randomized controlled trial to prevent excessive gestational weight gain-rationale and design of the GeliS study. BMC Pregnancy Childbirth 2014, 14, 119. [CrossRef] [PubMed]

26. Kunath, J.; Günther, J.; Rauh, K.; Hoffmann, J.; Stecher, L.; Rosenfeld, E.; Kick, L.; Ulm, K.; Hauner, H. Effects of a lifestyle intervention during pregnancy to prevent excessive gestational weight gain in routine careThe cluster-randomised GeliS trial. BMC Med. 2019, 17, 5. [CrossRef] [PubMed]

27. Günther, J.; Hoffmann, J.; Kunath, J.; Spies, M.; Meyer, D.; Stecher, L.; Rosenfeld, E.; Kick, L.; Rauh, K.; Hauner, H. Effects of a lifestyle intervention in routine care on prenatal dietary behavior-Findings from the cluster-randomized GeliS trial. J. Clin. Med. 2019. under revision.

28. Hoffmann, J.; Günther, J.; Geyer, K.; Stecher, L.; Rauh, K.; Kunath, J.; Meyer, D.; Sitzberger, C.; Spies, M.; Rosenfeld, E.; et al. Effects of a lifestyle intervention in routine care on prenatal physical activity-findings from the cluster-randomised GeliS trial. BMC Pregnancy Childbirth 2019. under revision. 
29. U.S. National Library of Medicine-ClinicalTrials.gov. Healthy Living in Pregnancy-NCT01958307. Available online: https://clinicaltrials.gov/ct2/show/NCT01958307 (accessed on 16 April 2019).

30. Koletzko, B.; Bauer, C.-P.; Bung, P.; Cremer, M.; Flothkötter, M.; Hellmers, C.; Kersting, M.; Krawinkel, M.; Przyrembel, H.; Rasenack, R.; et al. Practice recommendations of the Network "Healthy Start - Young Family Network". Dtsch. Med. Wochenschr. 2012, 137, 1366-1372. [CrossRef]

31. The American College of Obstetricians and Gynecologists. ACOG Committee Opinion No. 650: Physical Activity and Exercise During Pregnancy and the Postpartum Period. Obstet. Gynecol. 2015, 126, e135-42. [CrossRef]

32. Koletzko, B.; Bauer, C.-P.; Cierpka, M.; Cremer, M.; Flothkötter, M.; Graf, C.; Heindl, I.; Hellmers, C.; Kersting, M.; Krawinkel, M.; et al. Nutrition and physical activity of infants and breastfeeding women: Updated recommendations by "Healthy Start-Young Family Network" an initiative from IN FORM (German: Ernährung und Bewegung von Säuglingen und stillenden Frauen. Aktualisierte Handlungsempfehlungen von "Gesund ins Leben-Netzwerk Junge Familie", eine Initiative von IN FORM). Monatsschr Kinderheilkd 2016, 164, 771-798. [CrossRef]

33. Gunderson, E.P.; Abrams, B. Epidemiology of gestational weight gain and body weight changes after pregnancy. Epidemiol. Rev. 1999, 21, 261-275. [CrossRef]

34. Ohlin, A.; Rössner, S. Maternal body weight development after pregnancy. Int. J. Obes. 1990, 14, $159-173$. [PubMed]

35. Gunderson, E.P. Childbearing and obesity in women: Weight before, during, and after pregnancy. Obstet. Gynecol. Clin. North Am. 2009, 36, 317-332. [CrossRef] [PubMed]

36. Gore, S.A.; Della Brown, M.; West, D.S. The role of postpartum weight retention in obesity among women: A review of the evidence. Ann. Behav. Med. 2003, 26, 149-159. [CrossRef] [PubMed]

37. von der Lippe, E.; Brettschneider, A.-K.; Gutsche, J.; Poethko-Müller, C. Einflussfaktoren auf Verbreitung und Dauer des Stillens in Deutschland: Ergebnisse der KiGGS-Studie-Erste Folgebefragung (KiGGS Welle 1). Bundesgesundheitsblatt Gesundheitsforschung Gesundheitsschutz 2014, 57, 849-859. [CrossRef] [PubMed]

38. Topp, C.W.; Østergaard, S.D.; Søndergaard, S.; Bech, P. The WHO-5 Well-Being Index: A systematic review of the literature. Psychother. Psychosom. 2015, 84, 167-176. [CrossRef] [PubMed]

39. Bech, P.; Olsen, L.R.; Kjoller, M.; Rasmussen, N.K. Measuring well-being rather than the absence of distress symptoms: A comparison of the SF-36 Mental Health subscale and the WHO-Five Well-Being Scale. Int. J. Methods Psychiatr. Res. 2003, 12, 85-91. [CrossRef] [PubMed]

40. Horan, M.K.; McGowan, C.A.; Doyle, O.; McAuliffe, F.M. Well-being in pregnancy: An examination of the effect of socioeconomic, dietary and lifestyle factors including impact of a low glycaemic index dietary intervention. Eur. J. Clin. Nutr. 2014, 68, 19-24. [CrossRef] [PubMed]

41. Bergant, A.M.; Nguyen, T.; Heim, K.; Ulmer, H.; Dapunt, O. Deutschsprachige Fassung und Validierung der "Edinburgh postnatal depression scale". Dtsch. Med. Wochenschr. 1998, 123, 35-40. [CrossRef] [PubMed]

42. Donner, A.; Klar, N. Design and Analysis of Cluster Randomization Trials in Health Research; John Wiley \& Sons: Chichester, England, 2000; ISBN 0470711000.

43. Huang, T.-T.; Yeh, C.-Y.; Tsai, Y.-C. A diet and physical activity intervention for preventing weight retention among Taiwanese childbearing women: A randomised controlled trial. Midwifery 2011, 27, 257-264. [CrossRef] [PubMed]

44. Rauh, K.; Günther, J.; Kunath, J.; Stecher, L.; Hauner, H. Lifestyle intervention to prevent excessive maternal weight gain: Mother and infant follow-up at 12 months postpartum. BMC Pregnancy Childbirth 2015, 15, 265. [CrossRef] [PubMed]

45. Sagedal, L.R.; Sanda, B.; Øverby, N.C.; Bere, E.; Torstveit, M.K.; Lohne-Seiler, H.; Hillesund, E.R.; Pripp, A.H.; Henriksen, T.; Vistad, I. The effect of prenatal lifestyle intervention on weight retention 12 months postpartum: Results of the Norwegian Fit for Delivery randomised controlled trial. BJOG 2017, 124, 111-121. [CrossRef] [PubMed]

46. Ronnberg, A.; Hanson, U.; Ostlund, I.; Nilsson, K. Effects on postpartum weight retention after antenatal lifestyle intervention - a secondary analysis of a randomized controlled trial. Acta Obstet. Gynecol. Scand. 2016, 95, 999-1007. [CrossRef] [PubMed]

47. McCrabb, S.; Lane, C.; Hall, A.; Milat, A.; Bauman, A.; Sutherland, R.; Yoong, S.; Wolfenden, L. Scaling-up evidence-based obesity interventions: A systematic review assessing intervention adaptations and effectiveness and quantifying the scale-up penalty. Obes. Rev. 2019. [CrossRef] [PubMed] 
48. Hollis, J.L.; Crozier, S.R.; Inskip, H.M.; Cooper, C.; Godfrey, K.M.; Harvey, N.C.; Collins, C.E.; Robinson, S.M. Modifiable risk factors of maternal postpartum weight retention: An analysis of their combined impact and potential opportunities for prevention. Int. J. Obes. (Lond) 2017, 41, 1091-1098. [CrossRef] [PubMed]

49. Siega-Riz, A.M.; Viswanathan, M.; Moos, M.-K.; Deierlein, A.; Mumford, S.; Knaack, J.; Thieda, P.; Lux, L.J.; Lohr, K.N. A systematic review of outcomes of maternal weight gain according to the Institute of Medicine recommendations: Birthweight, fetal growth, and postpartum weight retention. Am. J. Obstet. Gynecol. 2009, 201, 339.e1-14. [CrossRef] [PubMed]

50. Hill, B.; McPhie, S.; Skouteris, H. The Role of Parity in Gestational Weight Gain and Postpartum Weight Retention. Womens. Health Issues 2016, 26, 123-129. [CrossRef] [PubMed]

51. Hill, B.; Bergmeier, H.; McPhie, S.; Fuller-Tyszkiewicz, M.; Teede, H.; Forster, D.; Spiliotis, B.E.; Hills, A.P.; Skouteris, H. Is parity a risk factor for excessive weight gain during pregnancy and postpartum weight retention? A systematic review and meta-analysis. Obes. Rev. 2017, 18, 755-764. [CrossRef] [PubMed]

52. Amorim, A.R.; Rössner, S.; Neovius, M.; Lourenço, P.M.; Linné, Y. Does excess pregnancy weight gain constitute a major risk for increasing long-term BMI? Obesity (Silver Spring) 2007, 15, 1278-1286. [CrossRef] [PubMed]

53. Ng, S.-K.; Cameron, C.M.; Hills, A.P.; McClure, R.J.; Scuffham, P.A. Socioeconomic disparities in prepregnancy BMI and impact on maternal and neonatal outcomes and postpartum weight retention: The EFHL longitudinal birth cohort study. BMC Pregnancy Childbirth 2014, 14, 314. [CrossRef]

54. Olson, C.M.; Strawderman, M.S.; Hinton, P.S.; Pearson, T.A. Gestational weight gain and postpartum behaviors associated with weight change from early pregnancy to 1 y postpartum. Int. J. Obes. Relat. Metab. Disord. 2003, 27, 117-127. [CrossRef]

55. Althuizen, E.; van der Wijden, C.L.; van Mechelen, W.; Seidell, J.C.; van Poppel, M.N.M. The effect of a counselling intervention on weight changes during and after pregnancy: A randomised trial. BJOG 2013, 120, 92-99. [CrossRef] [PubMed]

56. Robert Koch Institut. Journal of Health Monitoring | 3/2018 | KiGGS Welle 2-Gesundheitliche Lage. Available online: https:/www.rki.de/DE/Content/Gesundheitsmonitoring/Gesundheitsberichterstattung/ GBEDownloadsJ/JoHM_03_2018_KiGGS-Welle2_Gesundheitliche_Lage.pdf?_blob=publicationFile (accessed on 16 April 2019).

57. Henninger, M.L.; Irving, S.A.; Kauffman, T.L.; Kurosky, S.K.; Rompala, K.; Thompson, M.G.; Sokolow, L.Z.; Avalos, L.A.; Ball, S.W.; Shifflett, P.; et al. Predictors of Breastfeeding Initiation and Maintenance in an Integrated Healthcare Setting. J. Hum. Lact. 2017, 33, 256-266. [CrossRef] [PubMed]

58. Vieira, E.d.S.; Caldeira, N.T.; Eugênio, D.S.; Di Lucca, M.M.; Silva, I.A. Breastfeeding self-efficacy and postpartum depression: A cohort study. Rev. Lat. Am. Enfermagem 2018, 26, e3035. [CrossRef] [PubMed]

59. Amiel Castro, R.T.; Glover, V.; Ehlert, U.; O'Connor, T.G. Antenatal psychological and socioeconomic predictors of breastfeeding in a large community sample. Early Hum. Dev. 2017, 110, 50-56. [CrossRef] [PubMed]

60. Lara-Cinisomo, S.; McKenney, K.; Di Florio, A.; Meltzer-Brody, S. Associations Between Postpartum Depression, Breastfeeding, and Oxytocin Levels in Latina Mothers. Breastfeed. Med. 2017, 12, 436-442. [CrossRef] [PubMed]

61. Lederman, S.A.; Paxton, A. Maternal reporting of prepregnancy weight and birth outcome: Consistency and completeness compared with the clinical record. Matern. Child Health J. 1998, 2, 123-126. [CrossRef]

62. Tanentsapf, I.; Heitmann, B.L.; Adegboye, A.R.A. Systematic review of clinical trials on dietary interventions to prevent excessive weight gain during pregnancy among normal weight, overweight and obese women. BMC Pregnancy Childbirth 2011, 11. [CrossRef]

(C) 2019 by the authors. Licensee MDPI, Basel, Switzerland. This article is an open access article distributed under the terms and conditions of the Creative Commons Attribution (CC BY) license (http://creativecommons.org/licenses/by/4.0/). 